\title{
Development of a Model of the Infarcted Canine Heart that Predicts Arrhythmia Generation from Specific Cardiac Geometry and Scar Distribution
}

\author{
HJ Arevalo, PA Helm, NA Trayanova
}

Johns Hopkins University, Baltimore, MD, USA

\begin{abstract}
The role of the $3 D$ infarct structure in arrhythmia generation and maintenance in hearts with myocardial infarction cannot be fully elucidated through experimental techniques alone. Our aim was to develop methodology to construct a high resolution computational model of the infarcted canine heart using magnetic resonance (MRI) and diffusion tensor images (DTI) and then to use the resulting model to study postinfarction ventricular tachycardia (VT). Segmentation of the MRI and DTI stacks using image processing techniques provided accurate representations of the myocardium, the infarct scar, and the border zone (BZ) surrounding the scar. Realistic electrophysiological properties were assigned to each region. Simulations using the novel model revealed that postinfarction VT is initiated and maintained by reentrant circuits located within the BZ.
\end{abstract}

\section{Introduction}

Cardiac arrest remains the leading cause of death in the United States. Approximately two-thirds of these victims have a prior myocardial infarction (MI) [1]. Despite the disease's prevalence, the role of the complex infarct geometry and of the altered electrophysiology of the infarct zone in initiation and maintenance of ventricular tachycardia (VT) is not fully understood, hindering progress in the development of a reliable approach to ventricular ablation therapy.

Previous studies have reported that thin bands of viable myocardium positioned over intramural infarcts could act as the isthmus that anchors epicardial reentry [2]. These studies have utilized contrast-enhanced magnetic resonance imaging (MRI) in conjunction with surface electrical recordings to find correlations between surface activity and infarct geometries. Unfortunately, this technique is capable of providing insight into a subset of post-infarction VT only. Most VT induced in the clinical setting exhibit no surface reentry manifestations. This suggests that the complex architecture of the infarct within the ventricular wall plays a role in anchoring intramural reentry.

In addition to scar geometry, the electrical remodeling of cells near the infarct has been shown to promote VT initiation [3]. A border zone (BZ) of myocardium near the infarct experiences decreased perfusion which results in cellular electrical remodeling. While the BZ remains viable, it exhibits reduced excitability, longer action potential durations (APD), and redistribution of gap junction channels that result in altered conductivity. These remodeled cells facilitate VT induction by serving as sites of conduction block and reentry formation.

A major limitation that hinders the understanding of the role of infarct geometry and electrophysiology in arrhythmogenesis is the lack of experimental techniques that record transmural electrical activity with a high spatiotemporal resolution. Our previous work has demonstrated that validated realistic models of cardiac structure and electrical behavior can overcome this limitation [4]. The increase in computational power as well as the availability of high-resolution magnetic resonance imaging (MRI) and diffusion tensor (DT) MRI data sets have made possible the reconstruction of cardiac geometry and structure of large hearts, such as the dog or the human, and to use this geometrical and structural data to develop anatomically and electrophysiologically realistic models of these hearts.

The goals of this study are 1) to develop a set of tools that can be used to generate anatomically-accurate models of infarcted canine hearts based on high resolution MRI and DTMRI scans, and 2) to use the resulting models of infarcted canine hearts to explore and characterize post-MI VT formation and maintenance.

\section{Methods}

\subsection{Image-based model development}

A canine heart $\sim 4$ weeks post-infarction was scanned (MRI and DTMRI) at a resolution of $350 \times 350 \times 800 \mathrm{um}^{3}$ and interpolated, using splines, to a resolution of 200x200x200 $\mathrm{um}^{3}$ (Figure 1A). The first step in creating the model was the segmentation of the images to 
discriminate the myocardium from the surrounding suspension media. This was done by manually seeding points in the media and then using edge detection and region growing to create a mask. The tagged points in the mask were subtracted from the original image resulting in an accurate representation of the cardiac endocardium and epicardium. The cardiac segmentation was further refined by using a level-set method to ensure accurate delineation of myocardial microstructures from blood vessels within the organ.

A
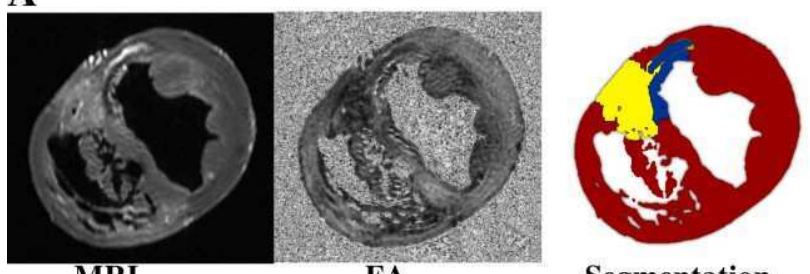

Segmentation

B

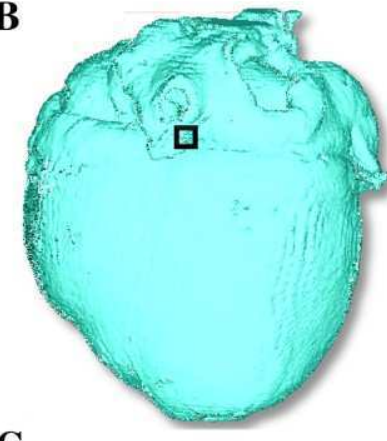

C
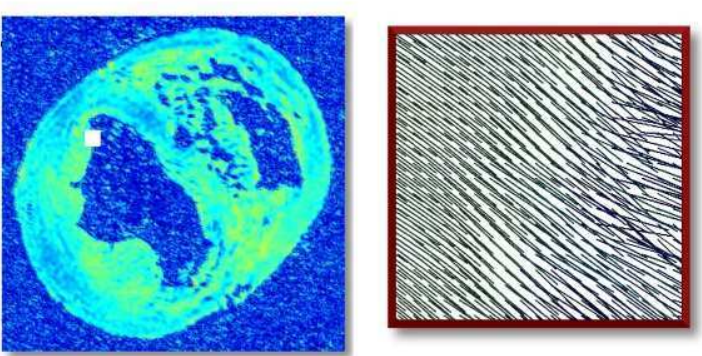

Figure 1. A) A representative MRI and FA slice and the segmented normal myocardium (red), border zone (yellow), and infarct scar (blue). B) Computational mesh of the heart, with black inset showing detail of the mesh. C) Map of primary eigenvectors with inset showing detail in area marked white. These vectors were used to represent fiber orientations in the heart.

Within the cardiac tissue, three distinct regions were further identified: the intact myocardium, and two regions within the infarct, an irreversibly injured central scar zone and a partially viable border zone (BZ). The regions of infarct were segmented by calculating the fractional anisotropy (FA) from the DTMRI data set (Figure 1A). The method was based on the fact that disorganized fiber orientation and an increased fluid volume within the infarct regions results in a lower FA compared to that of the normal myocardium. Points were manually seeded in the low FA region and a level-set method was used to automatically grow the seeded points into the infarct region. Gray-level thresholding of the MRI scan was subsequently used to distinguish between the two regions in the infarct.

The ventricles were then segmented from the atria through a semi-automatic approach. In every tenth slice in the saggital plane of the MRI stack, points were manually seeded to denote the atrioventricular border. Anatomical features such as the site of valves and differences in gray levels due to the presence of fibrous tissue were used to guide the seed point locations. These points were used to define a spline for the slice. The splines were then interpolated to automatically assign splines to the rest of the slices. These were used to fit a surface in 3D, representing the base of the ventricles.

With tissue segmentation complete, the commercial software Tarantula was used to create the finite element mesh of the heart and the surrounding volume (Figure 2B). The resulting mesh consisted of 5 million nodes and 29 million tetrahedral elements with an average edge length of 400um. This resolution was sufficient to represent structural details such as the infarct and the endocardial trabeculae, while keeping the model computationally manageable.

Finally, fiber orientations were assigned to each element of the mesh by calculating the primary eigenvectors of the diffusion tensors (Figure 1C).

\subsection{Electrophysiological properties}

The myocardium was assumed anisotropic. Normal conductivity values were used for the non-infarct tissue. $\mathrm{BZ}$ conductivity values were assigned based on previous studies reporting a $90 \%$ decrease in transverse coupling [3]. The infarct scar and atria were assumed nonexcitable.

The ionic kinetics in the normal and BZ myocardium were represented by the Luo-Rudy dynamic model [5]. Membrane kinetics in the BZ was modified based on data from literature. Previous studies of BZ in infarcted canine hearts have reported a reduction in peak sodium current to $38 \%$ of the normal value [6]; in peak L-type calcium current to $31 \%$ of normal [7]; and in peak potassium currents $I_{K r}$ and $I_{K s}$ to 30 and $20 \%$ of the maximum [8], respectively. These modifications result in longer APD and decreased excitability compared to the normal myocardium (Figure 2A). Mathematical description of current flow in cardiac tissue was based on the monodomain representation.

\subsection{Simulation Protocol}


To examine the arrhythmogenic propensity of the infarct substrate, an aggressive pacing protocol was delivered from six sites on the heart (Figure 2B), similar to protocols used for clinical evaluation of patients with MI. Pacing commenced at a cycle length of $250 \mathrm{~ms}$ for five beats (S1); 450ms after the last S1, 6 stimuli were delivered at progressively shorter coupling intervals, starting at $190 \mathrm{~ms}$ and decreasing in steps of $10 \mathrm{~ms}$. The induced activity was monitored for additional $2.5 \mathrm{~s}$.
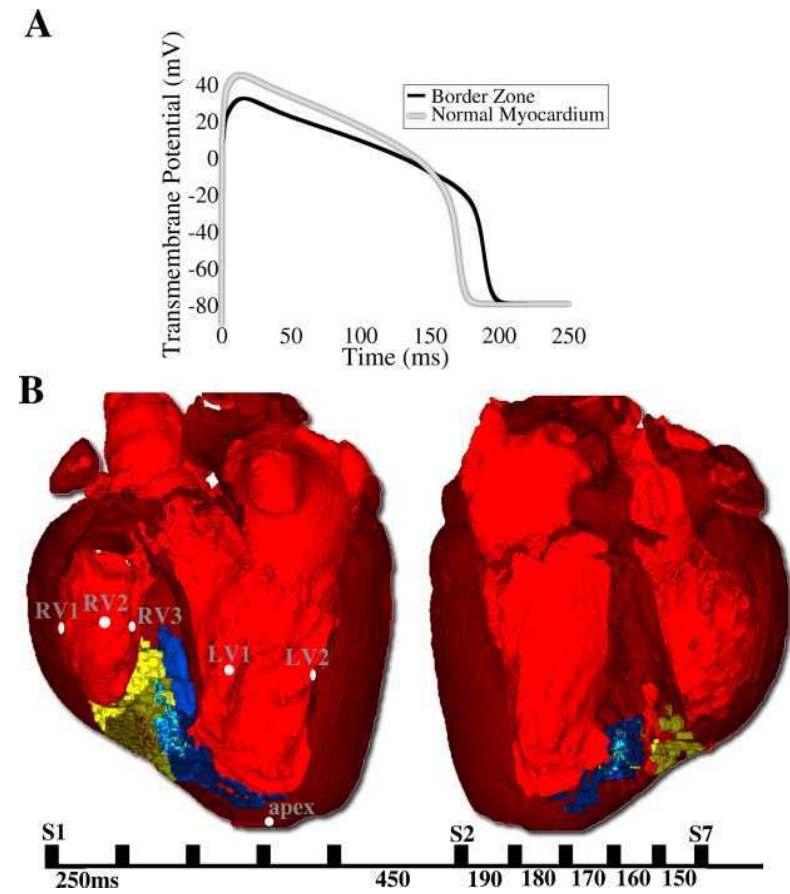

Figure 2. A) Action potentials in border zone and normal myocardium. B) Location of pacing sites in the heart and the pacing protocol (bottom) used to induce VT.

\section{Results}

After the successful image-based construction of the infracted canine heart model, simulations were conducted to explore VT initiation and its pattern. Aggressive pacing from each of the 6 sites resulted in successful induction of VT. Figure 3 illustrates the events that lead to VT induction in the case of apical pacing. Figure 3A depicts isochrones of epicardial activation times for the time period from the onset of a given stimulus (S4 or S6) to the time of delivery of the subsequent stimulus. Images on the right present the intramural activation pattern on the slice through the heart, the location of which is indicated by the white line on the epicardium. The white dotted line demarcates the BZ on the epicardial surface.

As indicated by the shorter distance between isochrones, conduction is significantly slower within the $\mathrm{BZ}$ as compared to the surrounding normal myocardium.
Fast wavefronts from the normal myocardium converge into the BZ laterally (white arrows) activating the entire BZ. Since the BZ has a longer APD, it remains refractory while the surrounding myocardium is fully recovered.
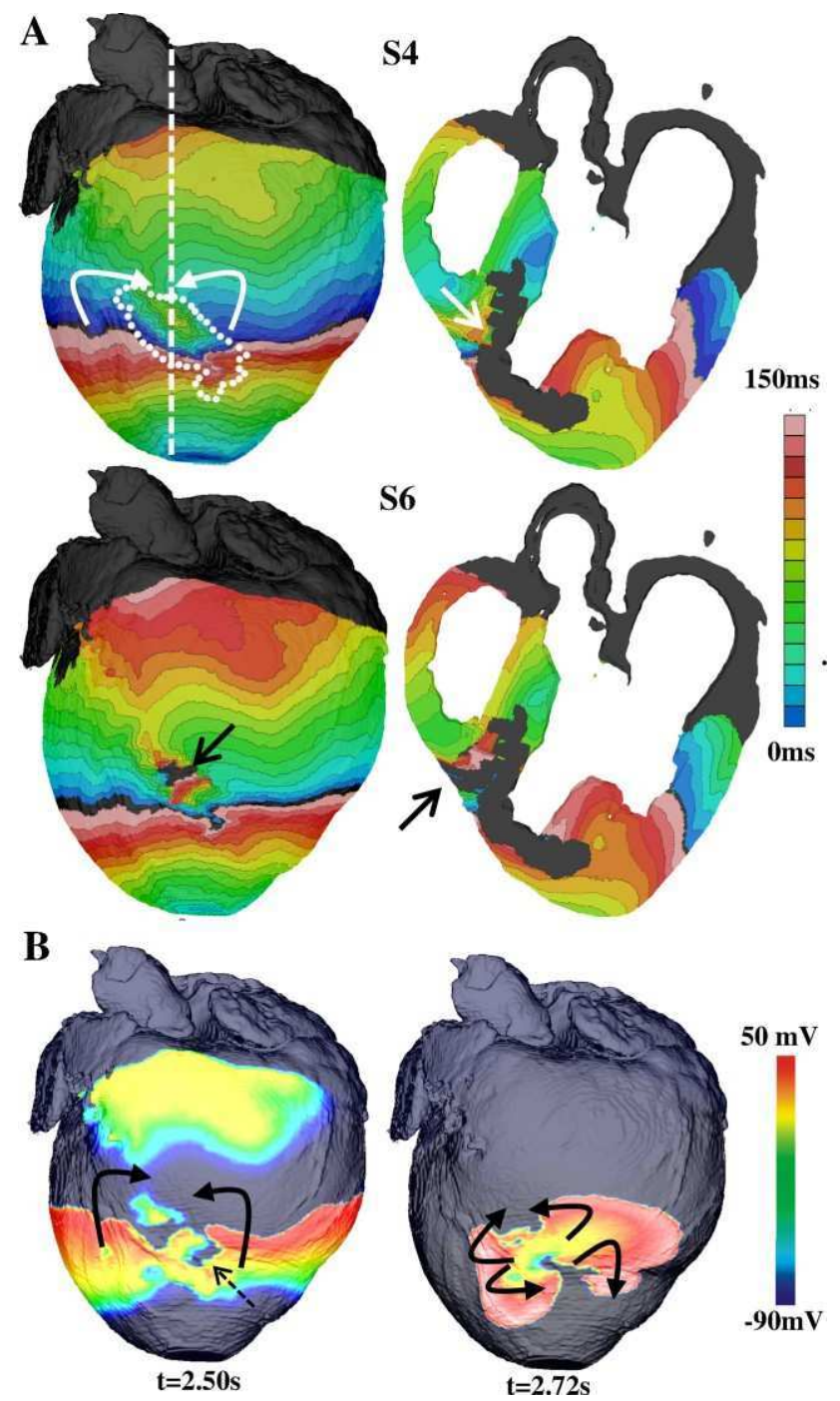

Figure 3. A) Activation maps for apical stimulation B) Transmembrane potential maps (see text for details).

At slow pacing rates, the refractory BZ does not result in reentry formation since it recovers prior to the arrival of the subsequent beat. As the pacing rate is increased, the wavefront encounters refractory tissue, resulting in conduction block. In Figure 3A, S6 panel, a sizeable portion of $\mathrm{BZ}$ does not activate because of conduction block of the preceding beat (black regions indicated by arrows), providing a pathway for reentry. Figure 3B shows corresponding transmembrane potential maps which demonstrate that at $\mathrm{t}=2.50 \mathrm{~s}$, wavefront propagates from the intramural BZ to the surface (dotted black 
arrow). When pacing is completed, the activation from within the BZ tissue develops into an epicardial quatrefoil reentry.

While the mechanism of VT initiation was similar for all pacing sites, the VT morphology varied for each simulation. VT ranged in frequency from 4.75 to $5.6 \mathrm{~Hz}$ with a mean of $5.155 \pm .35 \mathrm{~Hz}$. RV1 and LV2 pacing resulted in a figure-of- 8 reentrant pattern on the epicardium; for apical pacing the dominant pattern was epicardial quatrefoil reentry, and RV3 and LV1 pacing resulted in VT that switched between quatrefoil and figure-of- 8 epicardial reentry. In RV2 pacing, the reentrant source was not apparent from the surfaces.
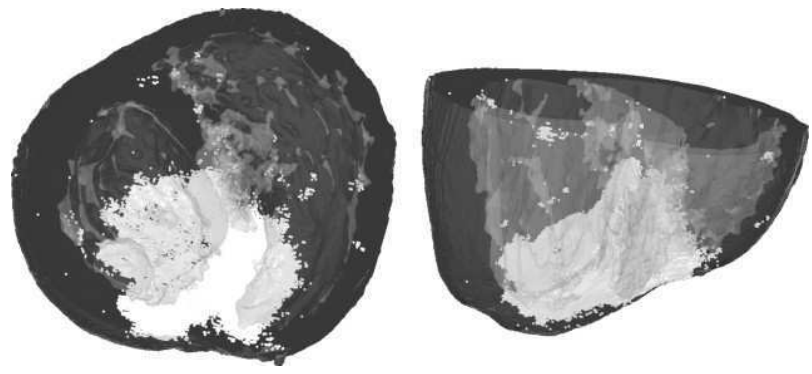

Figure 4. Regions where phase singularities reside. Elements with no PS formation are rendered transparent

The variety of VT morphologies is indicative of multiple sources of reentrant activity. The locations of phase singularities (PS), the organizing centers of reentry, are shown in Figure 4 for the six VT simulations. Tissue where no PS was formed is rendered transparent while sites of PS formations are colored white; the atria were removed for clarity. PS were overwhelmingly located within the posterior region of the heart, the location of the infarct. $76 \%$ of detected PS were located within $70 \%$ of the $\mathrm{BZ}$ volume. These results implicate the $\mathrm{BZ}$ as the main region of reentry formation and maintenance.

\section{Discussion and conclusions}

We successfully generated a high resolution model of canine heart that features accurate geometry, realistic fiber orientation, and anatomically based scar and BZ distribution, from a set of MRI and DTI scans. The methodologies developed for this study is readily applicable to further generation of large scale cardiac models. The resulting canine model was used to obtain novel insights into the role of infarct in arrhythmia generation and maintenance.

Our simulations show that the decreased excitability, longer APD, and reduced conduction velocity in the BZ promote conduction block and reentry formation. Although most of the VT manifested as epicardial reentry, investigation of intramural electrical activity reveals reentrant sources located within the BZ.
Our findings suggest that the frequent failure of catheter ablation of VT in clinical practice is due to the fact that the procedure relies on surface electrical recordings to determine the reentrant pathways and thus the ablation sites. Our results clearly demonstrate that the method is insufficient in characterizing the $3 \mathrm{D}$ extent of the infarction including the distribution of BZ tissue thus failing to identify all subsurface arrhythmogenic substrates. Clinical success rate can be greatly improved by developing techniques that address the intramural extent and composition of the infarct.

\section{References}

[1] Rothman S, Hsia H, Cossu S, et al. Radiofrequency catheter ablation of postinfarction ventricular tachycardia: long-term success and the significance of inducible nonclinical arrhythmias. Circ 1997; 96:3499-3508.

[2] Ashikaga H, Sasano T, Dong J, et al. Magnetic resonance based anatomical analysis of scar-related ventricular tachycardia. Implications for catheter ablation. Circ Res 2007; 101:1-9.

[3] Yao J-A, Hussain W, Patel P, et al. Remodeling of gap junctional channel function in epicardial border zone of healing canine infarcts. Circ Res 2003; 92:437-443.

[4] Arevalo H, Rodriguez B, Trayanova N. Arrhythmogenesis in the heart: Multiscale modeling of the effects of defibrillation shocks and the role of electrophysiological heterogeneity. Chaos 2007; 17:015103.

[5] Luo C-H, Rudy Y. A dynamic model of the cardiac ventricular action potential. Circ Res 1994; 74:1097-1113.

[6] $\mathrm{Pu}$ J, Boyden PA. Alterations of $\mathrm{Na}^{+}$currents in myocytes from epicardial border zone of the infarcted heart. A possible ionic mechanism for reduced excitability and postrepolarization refractoriness. Circ Res 1997; 81:110119.

[7] Dun W, Baba S, Yagi T, et al. Dynamic remodeling of $\mathrm{K}^{+}$ and $\mathrm{Ca}^{2+}$ currents in cells that survived in the epicardial border zone of canine healed infarcted heart. Am J Physiol Heart Circ Physiol 2004; 287:H1046-1054.

[8] Jiang M, Cabo C, Yao J, et al. Delayed rectifier K currents have reduced amplitudes and altered kinetics in myocytes from inarcted canine ventricle. Card Res 2000; 48:34-43.

Address for correspondence

Natalia Trayanova

3400 N. Charles St.

CSEB Room 216

Baltimore, MD 21218

ntrayanova@jhu.edu 